\title{
Exploring polycrystalline materials: high-throughput phase elucidation using serial rotation electron diffraction
}

\author{
Yi Luo, ${ }^{\# \dagger}$ Bin Wang, ${ }^{\sharp \dagger}$ Stef Smeets, ${ }^{\ddagger}$ Junliang Sun,,$*$ Weimin Yang,,${ }^{\S *}$ and Xiaodong Zou ${ }^{\dagger *}$
}

'Department of Materials and Environmental Chemistry, Stockholm University, SE-106 91 Stockholm, Sweden.

${ }^{\S}$ State Key Laboratory of Green Chemical Engineering and Industrial Catalysis, Sinopec Shanghai Research Institute of Petrochemical Technology, 1658 Pudong Beilu, Shanghai 201208, China.

Netherlands eScience Center, Science Park 140, 1098 XG Amsterdam, The Netherlands.

:College of Chemistry and Molecular Engineering, Beijing National Laboratory for Molecular Sciences, Peking University, Beijing 100871, China.

\#Y. Luo and B. Wang contributed equally to this work

*Corresponding authors: X. Z. (xiaodong.zou@mmk.su.se) and W. Y. (yangwm.sshy@sinopec.com).

\section{Abstract}

Rapidly and reliably elucidating the phases in polycrystalline materials is essential for developing new materials. Yet, crystals of many materials of biological, pharmaceutical, chemical, or industrial interest are too small $(<1$ $\mu \mathrm{m}$ ) for routine X-ray diffraction (XRD) analysis. For complex materials, this can result in workflow bottlenecks in high-throughput synthesis screenings favoured by industrial laboratories. With the increased prevalence of electron diffraction as an alternative technique for materials characterization, we explore a series of zeolite syntheses, resulting in typical polycrystalline products, via high-throughput phase identification using serial rotation electron diffraction (SerialRED). Five zeolite phases were identified in one product, the most complex mixture ever discovered in zeolite chemistry. Some of the phases are of ultra-low contents, similar unit cells, and/or identical morphologies. Via automatically examining hundreds of crystals, SerialRED enables the reliable and high-throughput phase analysis of products that XRD could not handle. It allows the exploration of more complex synthesis systems and provides new opportunities for rapidly developing novel polycrystalline materials, greatly benefiting synthesis chemistry and material science.

\section{Introduction}

Crystalline materials demonstrate unique optical, electrical, thermal, mechanical, and/or magnetic properties and have been extensively utilized. Nowadays, the synthesis conditions for exploring novel crystalline materials are becoming increasingly complex, motivated by growing and diverse demands. The resulting products are often formed as polycrystalline products ${ }^{1-3}$, and therefore difficult to study using routine X-ray diffraction methods. To unravel their phase compositions and atomic structures reliably at an early stage in the design of novel materials aids in rationalizing the synthesis, evaluating properties, and steering the design for promising new applications ${ }^{1,4-8}$. Meanwhile, recent trends of applying high-throughput synthesis screenings produce abundant polycrystalline products in a short time. Typical workflows require their phases to be identified and analysed at commensurate time scales to avoid bottlenecks ${ }^{9-16}$, but for polycrystalline materials this has remained a challenge ${ }^{17}$. 
Since its first discovery over a century ago, X-ray diffraction has been well-established for phase analysis and structure determination in chemistry. Single crystal X-ray diffraction (SCXRD) is the standard technique to obtain accurate crystal structures, as long as crystals are large enough (about $>5 \times 5 \times 5 \mu \mathrm{m} 3$ for a lab instrument) to harvest enough diffracted signal. Meanwhile, this being a single-crystal technique, makes phase analysis challenging. For this purpose, powder X-ray diffraction (PXRD) is routinely used, but it comes with its own set of limitations. Reflections with equal or similar $d$-spacings overlap in the one-dimensional pattern, making the phase and structure identification of polycrystalline materials by PXRD difficult and time-consuming, and sometimes impossible ${ }^{17-19}$. Challenges arise when a polycrystalline product contains 1) multiple phases, 2) phases with ultra-low contents $(<1 \%), 3)$ phases with similar unit cells, and/or 4) structures with large unit cells or low symmetries ${ }^{20}$. Some of the more interesting crystalline materials may therefore easily be ignored or overlooked. Electrons, on the other hand, diffract much more strongly. Compared to X-rays, the amount of observed diffraction signal using electron radiation is about six orders of magnitude higher per unit of volume, which enables useful data to be measured from crystals with sizes down to $50 \mathrm{~nm}^{20-24}$. Three-dimensional electron diffraction (3D ED) is analogous to SCXRD, but operates at a much smaller scale. The development of 3D ED methods has been rapid over the last decade, as researchers have discovered the benefits of this method to identify the phases and structures of polycrystalline materials that are too challenging to be studied by SCXRD/PXRD. The structures of many different types of polycrystalline materials have now been determined using 3D ED ${ }^{25-32}$. However, the searching of crystals for 3D ED data collection is still mostly a manual and time-consuming endeavour. The selection of crystal for data collection is therefore subject to human bias and some of the phases may be missed as a result. Although thousands of crystals are available on an electron microscopy grid, only a handful of crystals can be measured during a typical afternoon session. Therefore, we developed the highthroughput SerialRED method, which automatically screens crystals and collects 3D ED data, resulting in data from hundreds of crystals in a product ${ }^{33,34}$. It combines the best of SCXRD (structure determination) with PXRD (phase analysis) in a single technique. Combined with cluster analysis, SerialRED enables objective, highthroughput phase analysis and structure determination for multi-phasic submicron-sized crystal products (Scheme 1). This makes it an ideal technique to use in combination with the high-throughput synthesis screenings, helping us to accelerate the development of novel polycrystalline materials.

Here we demonstrate the application of SerialRED in the exploration of zeolites (Scheme 1). These are a class of typical metastable polycrystalline microporous materials that are widely applied in the industry ${ }^{35}$. Zeolites are often naturally composed of submicron-sized crystals with complex structures and frequently synthesized as multi-phases, which makes phase identification and structure analysis challenging ${ }^{17}$. In this work, we explore the combination of multiple framework $\mathrm{T}$ atoms $(\mathrm{T}=\mathrm{Si}, \mathrm{Ge}, \mathrm{Al}$, and $\mathrm{B})$ and a simple organic structure-directing agent (OSDA) to synthesize novel zeolite materials. We initially characterized the phase composition of the resulting 34 products by PXRD. Products that we could not identify with PXRD or manual 3D ED were then revealed by 35 SerialRED through high-throughput phase identification. This shows in practice the great advantage of 36 


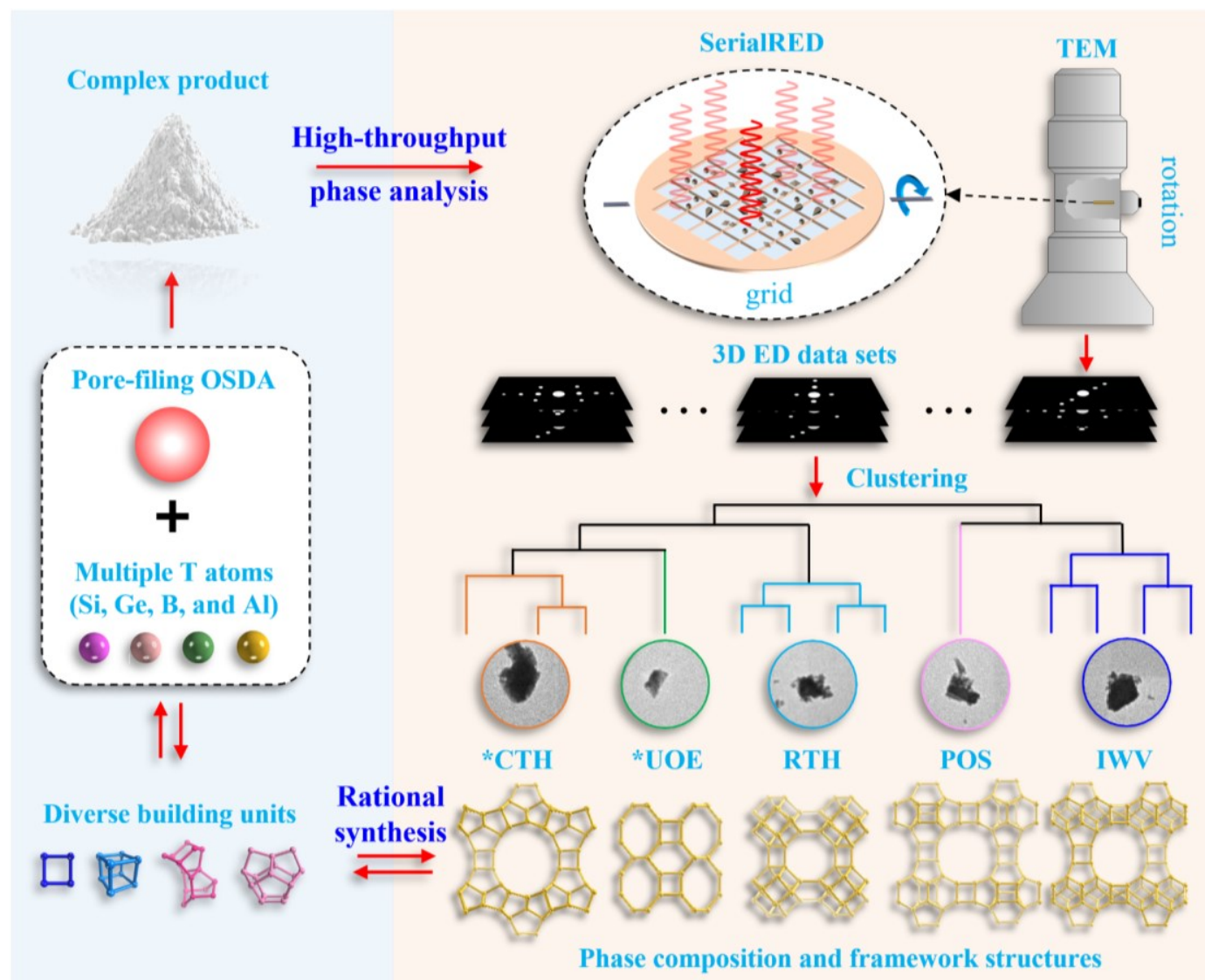

Scheme 1 Life cycle for exploring complex polycrystalline zeolite products via high-throughput phase identification using SerialRED. A complex synthesis system consisting of multiple framework T atoms and a single, simple OSDA was designed to synthesize zeolites. The different framework $\mathrm{T}$ atoms were expected to trigger the formation of a diverse variety of structure building units and result in various framework structures where the OSDA mainly plays a pore-filling role. The phase composition was studied by SerialRED. The cycle is completed by using the phase information in the rational development of novel zeolite materials.

\section{Results and discussion}

Synthesis and phase diagram. In Table 1, we show the lifecycle for exploring the synthesis of novel zeolite materials using two combinations of multiple framework $\mathrm{T}$ atoms ([Si,Ge,Al] and $[\mathrm{Si}, \mathrm{Ge}, \mathrm{B}]$ ) and a cheap, simple, commercially available pore filling OSDA (4-dimethylaminopyridine, DMAP). The OSDA has previously been used for the synthesis of zeolites with SFO, POS, and *UOE type framworks ${ }^{36-38}$. Studies have shown that the combination of framework $\mathrm{T}$ atoms directs the formation of specific structure building units ${ }^{39}$. Among them, $\mathrm{Si}$ and $\mathrm{Ge}([\mathrm{Si}, \mathrm{Ge}])$ are mostly combined together to synthesize large or extra-large pore zeolites that are normally unstable and lack active sites ${ }^{40}$. The addition of $\mathrm{Al}$ or $\mathrm{B}$ into the $[\mathrm{Si}, \mathrm{Ge}]$ system usually triggers an increase in the diversity of structure building units, introduce active sites, and result in thermal stable large or extra-large pore zeolites $^{39}$. In our synthesis, the $\mathrm{Si} / \mathrm{Ge}$ ratio was varied from 5 to 15 accompanied with the $(\mathrm{Si}+\mathrm{Ge}) / \mathrm{T}^{\mathrm{III}}\left(\mathrm{T}^{\mathrm{III}}=\mathrm{Al}\right.$ or B) ratios ranging from 0 to 100 to screen zeolite materials. Most synthesis batches give rise to crystalline products, including five pure phases and a series of mixtures of bi- or multi-phases (Table 1, S1, and S2). In the $[\mathrm{Si}, \mathrm{Ge}]$ system $\left((\mathrm{Si}+\mathrm{Ge}) \mathrm{T}^{\mathrm{III}=\infty}\right)$, two framework types, TON (1D, 10-ring) and POS (3D, 12×11×11-ring) were obtained (Table 1, S1, and S2) ${ }^{41}$. With the gradual introduction of B, framework types NON (0D) and SFE (1D, 
12-ring) were obtained (Table 1, S1, and S2), while the introduction of A1 triggers the formation of NON and RTH (2D, 8×8-ring) and a series of mixture products ${ }^{42-45}$.

Table 1 Synthesis parameters and phase diagrama ${ }^{\mathrm{a}}$.

\begin{tabular}{|c|c|c|c|c|}
\hline \multicolumn{5}{|c|}{$\mathrm{OSDA} / \mathrm{Si}=\mathbf{0 . 6}, \mathrm{HF} / \mathrm{Si}=\mathbf{0 . 6}, \mathrm{H}_{2} \mathrm{O} / \mathrm{Si}=\mathbf{1 0}$} \\
\hline & & \multicolumn{3}{|c|}{$\mathrm{Si} / \mathrm{Ge}$} \\
\hline & & 15 & 10 & 5 \\
\hline \multicolumn{2}{|c|}{$(\mathrm{Si}+\mathrm{Ge}) / \mathrm{T}^{\mathrm{III}}=\infty$} & TON & TON+POS & POS \\
\hline \multirow{5}{*}{$(\mathrm{Si}+\mathrm{Ge}) / \mathrm{Al}$} & 100 & NON & Amor. +Den.+*UOE & Amor.+Den.+*UOE \\
\hline & 20 & Amor. & Amor. + RTH+IWV+*CTH & RTH+*UOE+POS+IWV+*CTH \\
\hline & 15 & Amor.+Den.+*UOE+IWV & Den.+RTH+*UOE+POS+IWV+*CTH & RTH+*UOE + POS + IWV+*CTH \\
\hline & 10 & RTH & RTH+*UOE+POS+IWV+*CTH & RTH+IWV+*CTH \\
\hline & 5 & Amor. & Amor. & RTH \\
\hline \multirow{5}{*}{$(\mathrm{Si}+\mathrm{Ge}) / \mathrm{B}$} & 100 & NON & Amor. & Amor.+POS \\
\hline & 20 & Amor.+SFE & SFE & SFE+TON \\
\hline & 15 & Amor.+SFE & SFE & SFE+TON \\
\hline & 10 & SFE & SFE & SFE \\
\hline & 5 & SFE & SFE & SFE \\
\hline
\end{tabular}

${ }^{\mathrm{a}} \mathrm{T}^{\mathrm{III}}=\mathrm{Al}$ or $\mathrm{B}$, Amor.=Amorphous, Den.=Dense phase $\left(\mathrm{SiO}_{2} / \mathrm{GeO}_{2}\right)$. The phase information of mixture products that are extremely difficult or impossible to be identified by PXRD are highlighted in the bold black frame and green background.
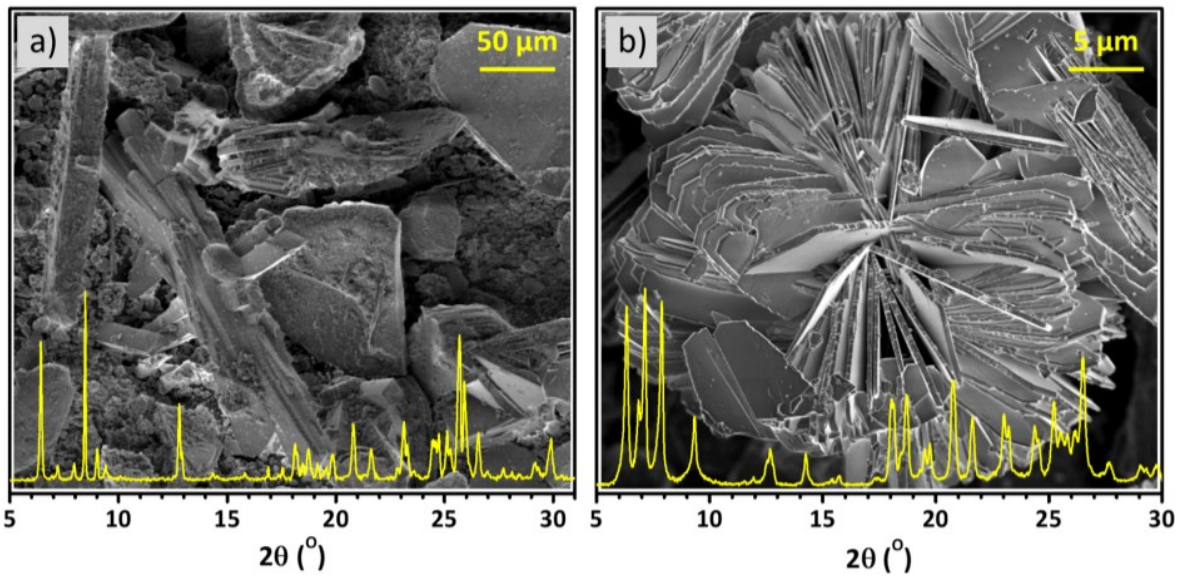

Figure 2 PXRD patterns (Cu Ka) and SEM images of (a) Product A and (b) B . Crystals with needle- and platelike morphologies are observed in Product $\mathrm{A}(\mathrm{Si} / \mathrm{Ge}=10,(\mathrm{Si}+\mathrm{Ge}) / \mathrm{Al}=15)$. Uniform plate-like crystals are presented in product $\mathrm{B}(\mathrm{Si} / \mathrm{Ge}=5,(\mathrm{Si}+\mathrm{Ge}) / \mathrm{Al}=12.5)$. The crystals were severely smashed into smaller pieces for the SerialRED

11 experiments.

The phase of these pure products, as well as most mixtures of bi-phases (with quite different unit cells or morphologies), were identified by PXRD with the assistance of scanning electron microscopy (SEM) (Figure S1 and S2). However, for some of the bi-phase products (with similar unit cells or morphologies) and most of the multi-phase products, only part of the phase information could be revealed by PXRD (Table 1). Figure 2a presents the PXRD pattern and SEM image of a typical complex mixture product (denoted as Product A). In Product A, only a significant RTH (needle-like) component was identified by PXRD (Figure S4a), but crystals with needleand plate-like morphologies were observed by SEM (Figure 2a). Therefore, RTH and an unknown phase with 
crystals, we used a two-step heating crystallization program in the synthesis to avoid the formation of RTH (see the synthesis details in supporting information). The obtained purified product (denoted as Product B) shows very high crystallinity and uniform plate-like crystals (Figure 2b and S5). Although the SEM images indicated that Product B is very likely to be a pure phase, we were unable to index its PXRD pattern using the SVD-index method implemented in Topas $V 6^{46}$.

Therefore, we turned to manual 3D ED, a technique that enables us to collect high-quality electron diffraction data for an isolated submicron-sized single-crystal. This revealed a zeolite IWV (2D, 12×12-ring, plate-like crystal) in Product B (Figure S6) ${ }^{5,47}$. Subsequently, IWV is also identified in Product A by comparing the PXRD patterns of Product A and B (Figure 2 and S4b). However, the PXRD pattern of Product B could only be indexed partially with the unit cell of IWV (Figure S7). When we tried to fit the PXRD pattern of Product A with two phases, RTH and IWV, we were also left with unindexed peaks (Figure S4b). Therefore, some of the phases in these two products may still be ignored by PXRD characterization and even by manual 3D ED study.

High-throughput phase identification using SerialRED. To systematically approach the problem and reveal all the phases in Product A and B, we used the SerialRED method that we developed in our $1 \mathrm{lab}^{33}$. SerialRED enables us to collect 3D ED data from hundreds of individual crystals automatically, with the aim to perform high-throughput phase analysis and structure determination. The SerialRED experiments were performed using the protocol implemented in the software Instamatic and on a trace amount of sample ${ }^{33,34}$. For Product A, the SerialRED routine ran for 6 hours resulting in 321 3D ED data sets (Figure S8). On our setup, we have integrated the program DIALS for on-the-fly unit cell identification for every data $\operatorname{set}^{24}$. As a result, 146 data sets were indexed with the corresponding unit cell parameters. Out of these, 77 out of 146 indexed data sets with a rotation range larger than $20^{\circ}$ were used for phase analysis. The high-throughput phase analysis was performed by feeding the identified unit cells into the hierarchical clustering analysis (HCA) algorithm implemented in the package edtools (Figure S9) 33,48 . The euclidean distance between the unit cell parameters was used as the metric for clustering.

Figure 3 and Table S1 present the clustering and phase analysis results of Product A. The cluster analysis revealed three more zeolite framework types, *UOE (2D, 10×8-ring, needle-like), POS (needle-like), and *CTH (2D, $14 \times 12$-ring, plate-like), in addition to RTH (needle-like) and IWV (plate-like) (Table S1, Table S2) ${ }^{49}$ that were already identified. RTH, IWV, and *CTH would be the major phases as indicated by the clustering results. The data sets in each cluster were merged, and the framework structures could be determined directly by SHELXT (Figure 3, Figure S10 and Table S3) ${ }^{33,50}$. These findings are corroborated by the Pawley fit of PXRD pattern of all phases using the routine implemented in the program TOPAS V6 ${ }^{46}$. The PXRD pattern of Product A can be well fitted with phases RTH, IWV, and ${ }^{*} \mathbf{C T H}$, which confirms that they are the major phases in Product A (Figure S4c). For *UOE and POS, which have morphologies almost identical to RTH (Figure 2), no obvious reflections from them were observed, and no significant improvement of the Pawley fit was obtained after including them (Figure S4c and S4d). This clearly highlights the great advantage of SerialRED in picking up minor phases that could not be detected by PXRD and/or SEM. 


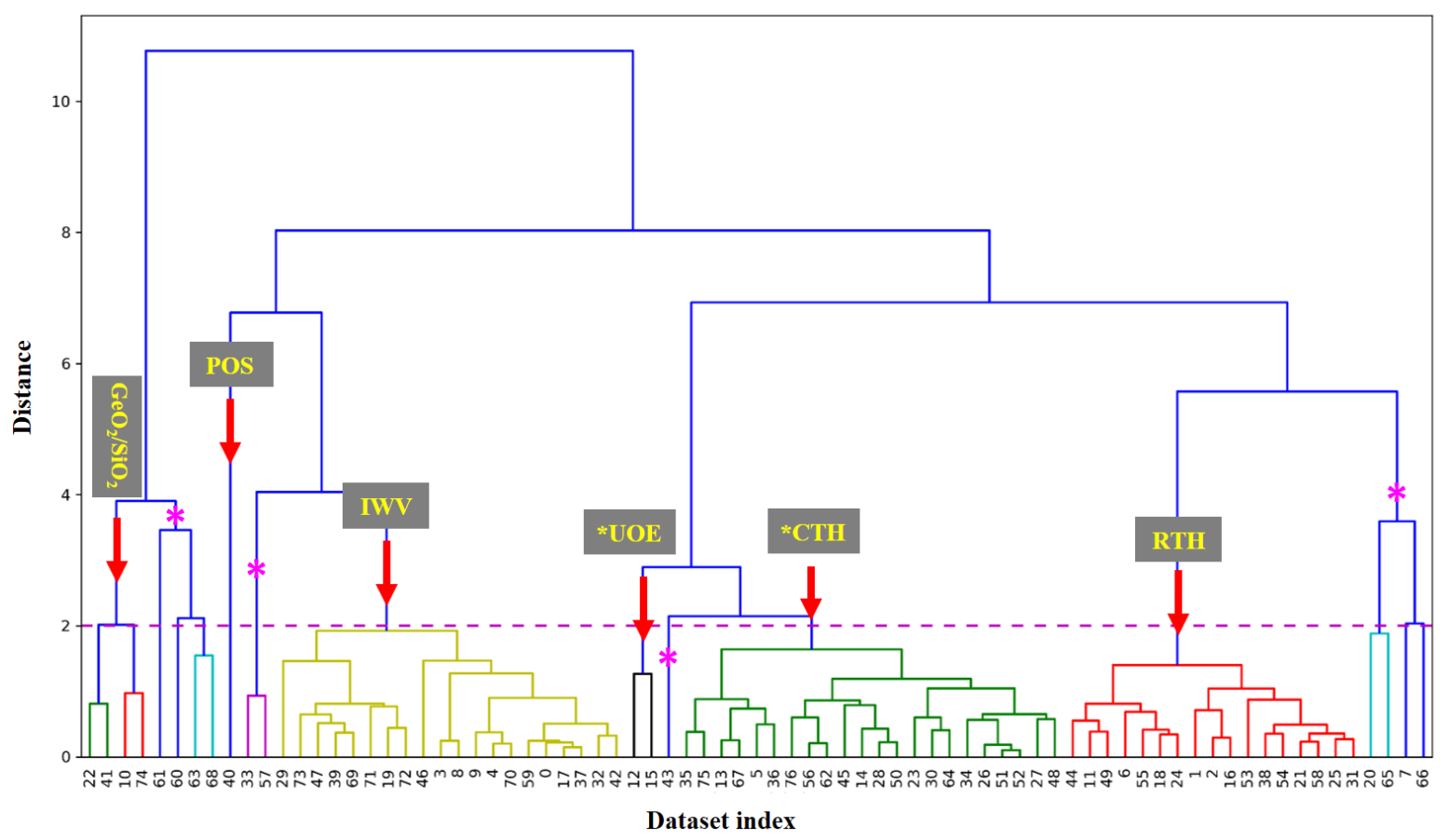

Figure 3 Dendrogram showing the results of the HCA of Product A. The y-axis is the euclidean distance between the unit cell parameters and is described in supporting information. HCA revealed five zeolite phases including RTH, IWV, *CTH, *UOE, and POS by setting the cut threshold at 2.0. Among them, RTH, IWV, and *CTH are the major phases. The unclassified data sets (marked by pink stars) could not be identified. These could be data from crystal agglomerations or of otherwise bad quality, which both result in inaccurate unit cell parameters with large deviations (Figure S11). The unit cell parameters of all data sets are shown in Table S1. In Product B, the phase ${ }^{*} \mathbf{C T H}$, which has a unit cell, morphology, and crystal size very similar to those of IWV, was also identified by SerialRED (Figure S12, S13, S14 and Table S4). Notably, we missed it in our initial assessment of the sample by using manual 3D ED, which can be attributed to a combination of small sampling size and crystal selection bias. The larger number of crystals sampled by SerialRED gives us a better statistical overview and thus more objective phase information of Product B. HCA of the SerialRED data revealed 75\% IWV : $25 \% *$ CTH from 36 crystals (counted based on the number of detected crystals, Table S4). Profile refinement of Product B against synchrotron powder X-ray diffraction (SPXRD) data using TOPAS V6 ${ }^{51}$ (Figure S15, Table S5) gave a phase composition of $67 \%$ IWV : $33 \% * \mathbf{C T H}$. The metrics differ in that SPXRD profile analysis determines weight percent and cluster analysis counts the number of crystals. Although our sampling size is still relatively small, this indicates that SerialRED is capable of quantitative phase analysis for crystalline materials as suggested previously ${ }^{33,52}$, as long as the number of crystals collected is large enough. SerialRED, the roles of different framework $\mathrm{T}$ atoms $\mathrm{Si}, \mathrm{Ge}, \mathrm{Al}$, and $\mathrm{B}$ in our synthesis system become clear. We noticed that the framework structures of NON, TON, SFE, IWV, and *CTH synthesized using the same OSDA (DMAP) are all highly related and containing similar chains (ton-, non-, $I W V$-, and $C T H$-chains) and layers (ton-, $n o n$-, $I W V$-, and $C T H$-layers) consisting of similar or identical building units (ton or $5^{2} 6^{2}$, Figure S16 and 4). Based on the common features in their framework structures and synthesis conditions, the formation of those chains, layers, or building units can be attributed to a Si-based system with a small amount of Ge (Figure S16 
and 4). Meanwhile, the differences in the framework structures and synthesis conditions show that the formation of non and $d 4 r$ units were triggered by a small amount of $\mathrm{B}$ or $\mathrm{Al}$ and a considerable amount of $\mathrm{Ge}$, respectively. Therefore, by introducing considerable Al and Ge into the synthesis system of TON, IWV and *CTH (both containing ton, non, and $d 4 r$ units) were formed (Figure 4). The close structural relationship between IWV and *CTH also explains the difficulty in synthesizing each in pure form. For SFE, its framework structure relates to that of TON through $\sigma$-expansion (Figure S17), which we achieved by introducing significant B into the synthesis system of TON. This clearly shows the role of B in promoting the formation of small $s 4 r$ units ${ }^{39}$. In our designed synthesis system, zeolite structures SFE, IWV, and ${ }^{*} \mathbf{C T H}$, which are prepared using bulky and expensive OSDAs (Table S6), were successfully synthesized using DMAP as OSDA. DMAP is simple, commercially available, and therefore economically more viable for the large-scale production of SFE, IWV, and ${ }^{*} \mathbf{C T H}$. In addition, our phase analysis results show that the two new zeolites * UOE and *CTH reported recently had already been synthesized in our system six years ago ${ }^{38,49,53}$. Were we able to identify these phases as novel at that time, we would have optimized our synthesis for those frameworks. Yet, we discarded the product as too complex to analyze. Therefore, we believe that SerialRED has a lasting place in our synthesis pipeline to identify interesting materials at an early stage of the synthesis. This approach is compatible with the way favoured by large-scale applications in industry, using SerialRED as a powerful and automated screening method for the exploration of such complex synthesis systems.

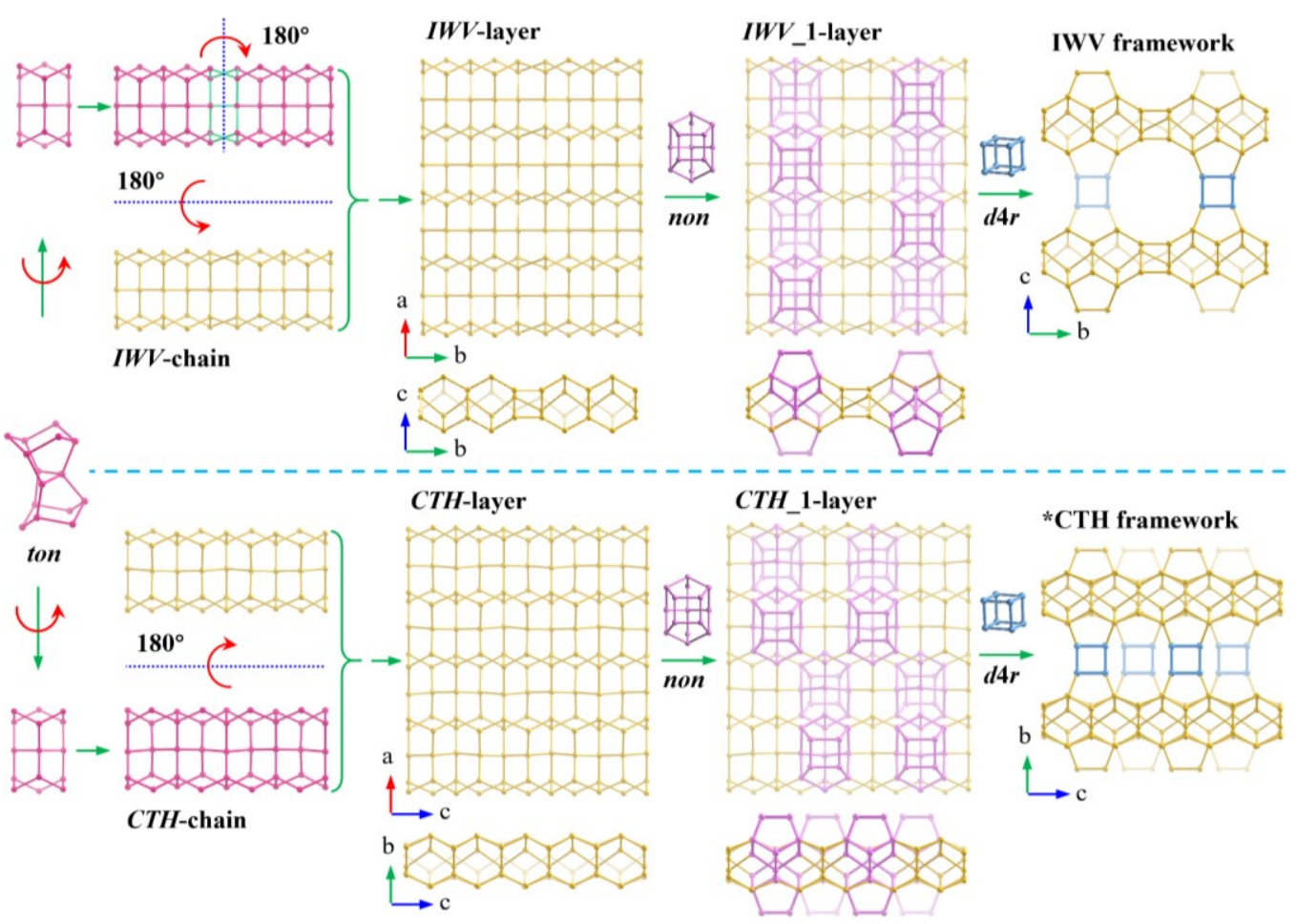

Figure 4 Structural relationship between IWV and ${ }^{*} \mathbf{C T H}$ (only one type of ordered frameworks was presented here). The frameworks of them are highly related and can both be constructed by ton, non, and $d 4 r$ building units in a very similar manner. The very similar $I W V$-layers and $C T H$-layers, which are also closely related to those of TON and NON (Figure S16), are built from ton units. The incorporation of non units on the $I W V$-layers and $C T H$-layers results in $I W V_{-} 1$-layers and $C T H_{-} 1$-layers, respectively. The neighbouring $I W V_{-} l$-layers and $C T H_{-} 1$-layers are then both connected by sharing $d 4 r$ units and forming IWV and $*$ CTH frameworks, respectively. 
Catalysis. In addition, the phase information also helps the understanding of the catalytic properties of Product B. The catalysis testing of product B was conducted on the isomerization of bulky isopropylnaphthalene molecules, which is a reaction to prepare important monomer 2,6-diisopropylnaphthalene for the advanced polyester fibers, films, and plastics (Figure S18-22 and Table S7, S8) ${ }^{54,55}$. Its performance was compared with those of MOR (2D, 12×12-ring) and SFE zeolites, which are excellent catalysts for the isomerization of isopropylnaphthalene ${ }^{55-58}$. Our results show that Product B with moderate acid properties has much higher catalytic efficiency than those of MOR and SFE (Figure S21, S22, and Table S8). This indicates that Product B has more accessible active sites for isopropylnaphthalene than those of MOR and SFE. This is because Product B contains 2D large-pore (IWV, 12×12-ring) and extra-large-pore $\left({ }^{*} \mathbf{C T H}, 14 \times 12\right.$-ring) zeolites, which have advantages in diffusing bulkier molecules. These offer us better ideas to develop the application of this catalyst in industrial processes that involve bulky molecules such as oil refining and fine chemical synthesis.

\section{Conclusions}

In this work, using the exploration of zeolite materials as a practical example, we demonstrated the great benefit of SerialRED in developing polycrystalline materials. Being able to automatically screen a large number of single crystals and collect 3D ED data, SerialRED offers new opportunities for rapidly accessing the reliable phase information of complex polycrystalline products via high-throughput screening. Five zeolites RTH, *UOE, POS, IWV, and *CTH, some of which were even with extra-low content, similar unit cells, and/or similar morphologies that were unable to be detected/identified by PXRD or even manual 3D ED, were revealed in the most complex zeolite mixture by SerialRED. The phase information helps to understand the roles of different framework $\mathrm{T}$ atoms and to accelerate the development of zeolite materials by being able to identify interesting phases at an early stage in the synthesis development. We also presented the possibility of the quantitative phase analysis using SerialRED data that showed general agreement with PXRD data. In addition, the SerialRED experiments are performed on a trace amount of sample, which is desirable for the nanomole-scale highthroughput synthesis chemistry. These advantages of SerialRED would essentially expand the scope of synthesis chemistry for interesting polycrystalline materials. Undoubtedly, apart from zeolite materials, SerialRED can also be a promising method with many future opportunities for facilitating the exploration of mineral, metal/metal oxide, ceramic, semiconductor, and also polymorphism in organic crystals including drugs, etc.

\section{References}

1. Guo, P. et al. A zeolite family with expanding structural complexity and embedded isoreticular structures. Nature 524, 74-78 (2015).

2. Collins, C. et al. Accelerated discovery of two crystal structure types in a complex inorganic phase field. Nature 546, 280-284 (2017).

3. Sun, W. et al. A map of the inorganic ternary metal nitrides. Nat. Mater. 18, 732-739 (2019).

4. Schwalbe-Koda, D. et al. A priori control of zeolite phase competition and intergrowth with high-throughput simulations. Science 374, 308-315(2021).

5. Gallego, E. M. et al. "Ab initio" synthesis of zeolites for preestablished catalytic reactions. Science 355, 1051-1054 (2017).

6. Bereciartua, P. J. et al. Control of zeolite framework flexibility and pore topology for separation of ethane and ethylene. Science 358, 1068-1071 (2017). 
7. Brand, S. K. et al. Enantiomerically enriched, polycrystalline molecular sieves. PNAS 114, 5101-5106 (2017).

8. Liu, X. et al. 3D Electron Diffraction Unravels the New Zeolite ECNU-23 from the "Pure" Powder Sample of ECNU-21. Angew. Chem. Int. Ed. 59, 1166-1170 (2020).

9. Corma, A., Díaz-Cabañas, M. J., Jordá, J. L., Martínez, C. \& Moliner, M. High-throughput synthesis and catalytic properties of a molecular sieve with 18- and 10-member rings. Nature 443, 842-845 (2006).

10. Banerjee, R. et al. High-Throughput Synthesis of Zeolitic Imidazolate Frameworks and Application to $\mathrm{CO}_{2} \mathrm{Capture}_{\text {. }}$ Science 319, 939-943 (2008).

11.Li, J. et al. Synthesis of many different types of organic small molecules using one automated process. Science 347, 1221-1226 (2015).

12.Gómez-Bombarelli, R. et al. Design of efficient molecular organic light-emitting diodes by a high-throughput virtual screening and experimental approach. Nat. Mater. 15, 1120-1127 (2016).

13. Raccuglia, P. et al. Machine-learning-assisted materials discovery using failed experiments. Nature 533, 73-76 (2016).

14. Steiner, S. et al. Organic synthesis in a modular robotic system driven by a chemical programming language. Science 363, 144 (2019).

15. Campos, K. R. et al. The importance of synthetic chemistry in the pharmaceutical industry. Science 363, 244 (2019).

16. Burger, B. et al. A mobile robotic chemist. Nature 583, 237-241 (2020).

17. Baerlocher, C. et al. Structure of the Polycrystalline Zeolite Catalyst IM-5 Solved by Enhanced Charge Flipping. Science 315, 1113-1116 (2007).

18.Gramm, F. et al. Complex zeolite structure solved by combining powder diffraction and electron microscopy. Nature 444, 79-81 (2006).

19. Sun, J. et al. The ITQ-37 mesoporous chiral zeolite. Nature 458, 1154-1157 (2009).

20.McCusker, L. \& Baerlocher, C. Electron crystallography as a complement to X-ray powder diffraction techniques. Z. Kristallogr. 228, 1-10 (2013).

21. Vainshtein, B. K. Structure Analysis by Electron Diffraction (Pergamon, 1964).

22.Dorset, D. L. \& Hauptman, H. A. Direct phase determination for quasi-kinematical electron diffraction intensity data from organic microcrystals. Ultramicroscopy 1, 195-201 (1976).

23. Dorset, D. L. Structural Electron Crystallography. (Springer, 1995).

24.Zou, X., Hovmöller, S. \& Oleynikov, P. Electron Crystallography: Electron Microscopy and Electron Diffraction. Electron Crystallography (Oxford University Press, 2011).

25.Gemmi, M. et al. Structure of $\mathrm{Ti}_{2} \mathrm{P}$ solved by three-dimensional electron diffraction data collected with the precession technique and high-resolution electron microscopy. Acta Cryst. A 59, 117-126 (2003).

26.Kolb, U., Gorelik, T., Kübel, C., Otten, M. T. \& Hubert, D. Towards automated diffraction tomography: Part I-Data acquisition. Ultramicroscopy 107, 507-513 (2007).

27.Zhang, D., Oleynikov, P., Hovmöller, S. \& Zou, X. Collecting 3D electron diffraction data by the rotation method. Z. Kristallogr. 225, 94-102 (2010).

28.Shi, D., Nannenga, B. L., Iadanza, M. G. \& Gonen, T. Three-dimensional electron crystallography of protein microcrystals. eLife 2, e01345 (2013).

29.Nederlof, I., van Genderen, E., Li, Y.-W. \& Abrahams, J. P. A Medipix quantum area detector allows rotation electron diffraction data collection from submicrometre three-dimensional protein crystals. Acta Cryst. D 69, 12231230 (2013).

30.Yun, Y. et al. Phase identification and structure determination from multiphase crystalline powder samples by rotation electron diffraction. J. Appl. Cryst. 47, 2048-2054 (2014).

31.Palatinus, L. et al. Hydrogen positions in single nanocrystals revealed by electron diffraction. Science 355, 166169 (2017).

32.Gemmi, M. et al. 3D Electron Diffraction: The Nanocrystallography Revolution. ACS Cent. Sci. 5, 1315-1329 (2019).

33.Wang, B., Zou, X. \& Smeets, S. Automated serial rotation electron diffraction combined with cluster analysis: an efficient multi-crystal workflow for structure determination. IUCrJ 6, 854-867 (2019). 
34.Cichocka, M. O., Ångström, J., Wang, B., Zou, X. \& Smeets, S. High-throughput continuous rotation electron diffraction data acquisition via software automation. J. Appl. Cryst. 51, 1652-1661 (2018).

35.Davis, M. E. Ordered porous materials for emerging applications. Nature 417, 813-821 (2002).

36. Morris, R. E., Burton, A., Bull, L. M. \& Zones, S. I. SSZ-51-A New Aluminophosphate Zeotype: Synthesis, Crystal Structure, NMR, and Dehydration Properties. Chem. Mater. 16, 2844-2851 (2004).

37.Hua, W. et al. A Germanosilicate Structure with $11 \times 11 \times 12$-Ring Channels Solved by Electron Crystallography. Angew. Chem. Int. Ed. 53, 5868-5871 (2014).

38. Cichocka, M. O. et al. Multidimensional Disorder in Zeolite IM-18 Revealed by Combining Transmission Electron Microscopy and X-ray Powder Diffraction Analyses. Cryst. Growth Des. 18, 2441-2451 (2018).

39.Zones, S. I. Translating new materials discoveries in zeolite research to commercial manufacture. Microporous Mesoporous Mater. 144, 1-8 (2011).

40.Li, J., Corma, A. \& Yu, J. Synthesis of new zeolite structures. Chem. Soc. Rev. 44, 7112-7127 (2015).

41.Barri, S. a. I., Smith, G. W., White, D. \& Young, D. Structure of Theta-1, the first unidimensional medium-pore high-silica zeolite. Nature 312, 533-534 (1984).

42.Marler, B., Dehnbostel, N., Eulert, H.-H., Gies, H. \& Liebau, F. Studies on clathrasils VIII. Nonasils-[4 $\left.{ }^{1} 5^{8}\right]$, $88 \mathrm{SiO}_{2} \cdot 8 \mathrm{M}^{8} \cdot 8 \mathrm{M}^{9} \cdot 4 \mathrm{M}^{20}$ : Synthesis, thermal properties, and crystal structure. J. Incl. Phenom. 4, 339-349 (1986).

43. Vortmann, S., Marler, B., Gies, H. \& Daniels, P. Synthesis and crystal structure of the new borosilicate zeolite RUB-13. Microporous Mater. 4, 111-121 (1995).

44.Lee, G. S. et al. Organocations in Zeolite Synthesis: Fused Bicyclo [I.m.0] Cations and the Discovery of Zeolite SSZ-48. J. Am. Chem. Soc. 124, 7024-7034 (2002).

45.Baerlocher, C. \& McCusker, L. B. Database of Zeolite Structures. http://europe.iza-structure.org/IZA-SC.

46. Coelho, A. A. TOPAS and TOPAS-Academic: an optimization program integrating computer algebra and crystallographic objects written in C++. J. Appl. Cryst. 51, 210-218 (2018).

47.Dorset, D. L. et al. P-Derived Organic Cations as Structure-Directing Agents: Synthesis of a High-Silica Zeolite (ITQ-27) with a Two-Dimensional 12-Ring Channel System. J. Am. Chem. Soc. 128, 8862-8867 (2006).

48. Smeets, S. https://github.com/instamatic-dev/edtools.

49.Kang, J. H. et al. Synthesis and Characterization of CIT-13, a Germanosilicate Molecular Sieve with Extra-Large Pore Openings. Chem. Mater. 28, 6250-6259 (2016).

50.Sheldrick, G. M. SHELXT - Integrated space-group and crystal-structure determination. Acta Cryst. A 71, 3-8 (2015).

51.Smeets, S. et al. Locating Organic Guests in Inorganic Host Materials from X-ray Powder Diffraction Data. J. Am. Chem. Soc. 138, 7099-7106 (2016).

52.Smeets, S., Ångström, J. \& Olsson, C.-O. A. Quantitative Phase Analysis for Carbide Characterization in Steel Using Automated Electron Diffraction. Steel Res. Int. 90, 1800300 (2019).

53. Yang, W., Wang, Z., Sun, H., Zhang, B. \& Luo, Y. SCM-11 molecular sieve, process for producing same and use thereof. (2019).

54.Song, C. \& Schobert, H. H. Opportunities for developing specialty chemicals and advanced materials from coals. Fuel Process. Technol. 34, 157-196 (1993).

55.Brzozowski, R. \& Skupiński, W. Disproportionation of isopropylnaphthalene on zeolite catalysts. J. Catal. 220, 13-22 (2003).

56.Schmitz, A. D. \& Song, C. Shape-selective isopropylation of naphthalene. Reactivity of 2,6-diisopropylnaphthalene on dealuminated mordenites. Catal. Today 31, 19-25 (1996).

57.Brzozowski, R. \& Buijs, W. Shape-selective synthesis of 2,6-diisopropylnaphthalene on H-mordenite catalysts. $J$. Catal. 292, 181-187 (2012).

58.Luo, Y. et al. A Facile and Green Method for the Synthesis of SFE Borosilicate Zeolite and Its HeteroatomSubstituted Analogues with Promising Catalytic Performances. Chem. Eur. J. 24, 306-311 (2018). 
1 The authors gratefully acknowledge the beamline scientists (beamline BL14B1 at the SSRF, Shanghai, China)

2 for their assistance with the SPXRD experiments. The authors acknowledge financial support from the Swedish 3 Research Council (VR, 2017-04321; 2019-00815), the Knut and Alice Wallenberg Foundation (KAW, 4 2012.0112, 2018.0237), the National Key R\&D Program of China (2017YFB0702800), and the China

5 Petrochemical Corporation (Sinopec Group).

6 Author contributions

$7 \quad$ X. Z. directed the SerialRED study. W. Y. directed the synthesis of zeolites. Y. L. designed and performed the 8 synthesis experiments of zeolites. B. W. and Y. L. performed the SerialRED data collection and analysis. B. W. 9 and S. S. developed the SerialRED method. B. W. and Y. L. improved the data processing of SerialRED. B. W.

10 conducted the structure refinement against the SerialRED data. Y. L. performed the Rietveld refinement, Pawley 11 fit, catalytic study, and all other characterizations. J. S. supported the manual 3D ED experiments on the phase 12 identification of $\mathbf{I W V}$ in product B. Y. L., B. W., and S. S. wrote the initial draft. All authors reviewed and 13 commented on the manuscript.

\section{Competing interests}

15 The authors declare no competing interests. 